Effect of acetyl-I-carnitine in the treatment of diabetic peripheral neuropathy : A systematic review and meta-analysis

\title{
Veronese, N.
}

2017-04

Veronese , N, Sergi , G , Stubbs , B , Bourdel-Marchasson , I, Tessier , D , Sieber , C , Strandberg, T, Gillain , S, Barbagallo, M , Crepaldi , G, Maggi , S, Manzato , E \& EUGMS Special Interest Grp Diabet 2017 , ' Effect of acetyl-I-carnitine in the treatment of diabetic peripheral neuropathy : A systematic review and meta-analysis ' , European Geriatric Medicine , vol. 8 , no. 2 , pp. 117-122 . https://doi.org/10.1016/j.eurger.2017.01.002

http://hdl.handle.net/10138/235591

https://doi.org/10.1016/j.eurger.2017.01.002

unspecified

publishedVersion

Downloaded from Helda, University of Helsinki institutional repository.

This is an electronic reprint of the original article.

This reprint may differ from the original in pagination and typographic detail.

Please cite the original version. 


\title{
Effect of acetyl-l-carnitine in the treatment of diabetic peripheral neuropathy: A systematic review and meta-analysis
}

\author{
N. Veronese ${ }^{a, *}, G$. Sergi ${ }^{a}$, B. Stubbs ${ }^{\text {b,c,d }}$, the EUGMS special interest group on diabetes, \\ I. Bourdel-Marchasson ${ }^{\mathrm{e}, \mathrm{f}}$, D. Tessier ${ }^{\mathrm{g}}$, C. Sieber ${ }^{\mathrm{h}}$, T. Strandberg ${ }^{\mathrm{i}, \mathrm{j}}$, S. Gillain ${ }^{\mathrm{k}}$, \\ M. Barbagallo ${ }^{1}$, G. Crepaldi ${ }^{\mathrm{m}}$, S. Maggi ${ }^{\mathrm{m}}$, E. Manzato ${ }^{\mathrm{a}}$ \\ ${ }^{a}$ University of Padova, department of medicine, geriatrics section, via Giustiniani, 2, 35128 Padova, Italy \\ ${ }^{\mathrm{b}}$ Physiotherapy department, South London and Maudsley National Health Service Foundation Trust, London, United Kingdom \\ ${ }^{\mathrm{c}}$ Health service and population research department, institute of psychiatry, psychology and neuroscience, King's College London, London, United Kingdom \\ ${ }^{\mathrm{d}}$ Faculty of health, social care and education, Anglia Ruskin university, Chelmsford, United Kingdom \\ e Clinical gerontology department, CHU de Bordeaux, 33000 Bordeaux, France \\ ${ }^{\mathrm{f}}$ RMSB, UMR 5536, CNRS, 33400 Bordeaux, France \\ ${ }^{\mathrm{g}}$ Geriatric division, Sherbrooke university, Quebec, Canada \\ ${ }^{\mathrm{h}}$ Institute for biomedicine of aging, Friedrich-Alexander-Universität Erlangen-Nürnberg, Nuremberg, Germany \\ ${ }^{\mathrm{i}}$ Geriatric clinic, department of medicine, university of Helsinki, Helsinki, Finland \\ ${ }^{\mathrm{j}}$ Institute of health sciences/geriatrics, university of Oulu, Oulu university hospital, Oulu, Finland \\ ${ }^{\mathrm{k}}$ Service de gériatrie, $\mathrm{CHU}$ de Liège, Liège, Belgium \\ ${ }^{1}$ Geriatric unit, department of internal medicine and geriatrics, university of Palermo, Palermo, Italy \\ ${ }^{\mathrm{m}}$ National research council, aging branch neuroscience institute, Padova, Italy
}

\section{A R T I C L E I N F O}

\section{Article history:}

Received 16 November 2016

Accepted 8 January 2017

Available online 28 January 2017

Keywords:

Acetyl-L-carnitine

Neuropathy

Diabetes

Meta-analysis

\begin{abstract}
A B S T R A C T
Background/aim: Deficiency of acetyl-L-carnitine (ALC) and L-carnitine (LC) appears to play a role in peripheral diabetic neuropathy, although the evidence in humans is still limited. We conducted a systematic review and meta-analysis investigating the effect of ALC on pain and electromyographic parameters in people with diabetic neuropathy.

Methods: A literature search in major databases, without language restriction, was undertaken. Eligible studies were randomized controlled trials (RCTs) or pre- and post-test studies. The effect of ALC supplementation on pain perception and electromyographic parameters in patients with diabetic neuropathy was compared vs. a control group (RCTs). The effect of ALC/LC on electromyographic parameters were also calculated vs. baseline values. Standardized mean differences (SMD) and 95\% confidence intervals (CIs) were used for summarizing outcomes.

Results: Six articles, with a total of 711 diabetic participants, were included. Three RCTs ( 340 treated with ALC vs. 203 placebo and 115 with methylcobalamine) showed that ALC reduces pain perception ( $\mathrm{SMD}=-0.45 ; 95 \% \mathrm{CI}:-0.86$ to $-0.04 ; P=0.03 ; \mathrm{I}^{2}=85 \%$ ). Compared to controls, ALC supplementation improved nerve conduction velocity and amplitude response for ulnar nerve (both sensory and motor component). Compared to baseline values, ALC/LC supplementation improved nerve conduction velocity for all the sensory and motor nerves (except ulnar and peroneal) investigated and the amplitude of all nerves. The onset of adverse events was generally limited to minor side effects.

Conclusion: ALC appears to be effective in reducing pain due to diabetic neuropathy compared to active or placebo controls and improving electromyographic parameters in these patients.
\end{abstract}

(c) 2017 Elsevier Masson SAS and European Union Geriatric Medicine Society. All rights reserved.

\section{Introduction}

Peripheral neuropathy is one of the most prevalent complications of diabetes mellitus, with prevalence estimates ranging from

\footnotetext{
* Corresponding author. Tel.: +00390498218492; fax: +00390498211218.

E-mail address: ilmannato@gmail.com (N. Veronese).
}

12 to $50 \%$ [1]. The most common form of peripheral neuropathy in diabetes is the distal symmetric polyneuropathy, a form affecting both sensory and motor nerves [1].

The complications due to diabetic neuropathy are multiple (e.g. asthenia, paresthesia in initial stages and in advanced ones ulcers and deformation of lower extremities) and often associated with higher presence of disability and poor quality of life [2]. These 
effects are mainly due to the presence of pain, one of the most common symptoms associated with diabetic neuropathy [1].

The management of symptomatic diabetic sensory neuropathy presents a therapeutic challenge for the physicians, since the therapies for this conditions are predominantly limited to seeking to obtain good glucose control and the use of analgesic medications $[3,4]$. Such interventions are often not effective in symptoms and do not address the pathogenesis of diabetic neuropathy. Although the pathogenesis of this condition is not completely understood, one of the main mechanisms responsible seems to be the reduced availability of acetyl groups, necessary for the synthesis of choline [5]. A deficiency of acetyl-L-carnitine (ALC) and L-carnitine (LC) seems to play a pivotal role in this pathway [6], since a deficit of ALC has been shown to cause damage to the myelin sheath [7]. In animal models, the exogenous administration of ALC increases artemin levels and enhances the expression of nerve growth factor (NGF) $[8,9]$, enhances antioxidant activity [10] and microvascular protein permeability [11], and induces long-term upregulation of the presynaptic mGlu2 receptors [12]; in this way, ALC supplementation induces neuroprotective, neurotrophic and analgesic effects in the peripheral nervous system $[12,13]$.

Regarding human beings, ALC is recommended in the tier 1 of neuropathic pain treatments by the Mayo Clinic proceedings [14], and a recent meta-analysis has confirmed that administration of ALC was able to improve pain perception in people affected by peripheral neuropathy [15]. Although this work advanced our knowledge regarding this important topic, a number of limitations persist, for instance, the authors considered all kinds of neuropathies together (although they have different pathogeneses) and they did not investigate the effect of ALC on electromyographic parameters, the most common method for diagnosis and evaluating peripheral neuropathy [1].

Given the mentioned limitations in the literature, we aimed to investigate the effect of ALC on pain and electromyographic parameters in diabetic neuropathy. We hypothesized that ALC supplementation is beneficial for symptomatology and electromyographic features of diabetic neuropathy.

\section{Methods}

This systematic review adhered to the Prisma [16] and Moose [17] statements and followed a structured, but unpublished protocol.

\subsection{Data sources and literature search strategy}

Two investigators (NV and GS) independently conducted a literature search using PubMed, Embase, Scopus, Cochrane central register of controlled trials and Clinicaltrials.gov without language restriction, from database inception until 5th June 2016 for randomized controlled trials (RCTs) and descriptive studies (i.e. without a control group) investigating the effect of ALC supplementation in patients with diabetic neuropathy.

In PubMed, the following search strategy was used: "(carnitine [Text word] OR "Carnitine"[Mesh]) AND (diabet*) AND (neurop*)". Conference abstracts and reference lists of included articles were hand-searched to identify and potential additional relevant articles. Any inconsistencies were resolved by consensus with a third author (SM).

\subsection{Study selection}

Inclusion criteria for this meta-analysis were:

- RCTs or pre- or post-test studies;

- included diabetic participants with peripheral neuropathy diagnosed through electromyography (EMG);
- investigated the use of carnitine supplementation (regardless of administration route);

- included data regarding pain (due to neuropathy) and/or regarding EMG parameters.

Studies were excluded if:

- did not include humans;

- investigated the effect of carnitine on other causes of neuropathic pain (e.g. due to chemotherapy);

- included participants with diabetes, but without a neuropathy.

\subsection{Data extraction}

Two independent investigators (NV and BS) extracted key data from the included articles in a standardized Excel sheet. A third independent investigator (GS) checked the extracted data.

For each article, we extracted data about authors, year of publication, country, study design (RCT/descriptive), medications used for the treatment of diabetes, daily ALC/LC dosage, follow-up duration (in weeks) and mean age (by treatment type: ALC or control group). Finally, we extracted data regarding the adverse events reported in each study.

\subsection{Outcomes}

The primary outcome was the change of pain perception at follow-up assessed through a validated scale (e.g. Visual Analogue Scale) between participants treated with ALC vs. controls.

As secondary outcomes, we considered EMG parameters in terms of conduction velocity and response amplitude in terms of:

- differences between follow-up and baseline (pre and post treatment) in ALC vs. controls;

- within patients treated comparing the data at follow-up vs. baseline in people treated with ALC/LC.

\subsection{Assessment of study quality}

Two authors (NV, GS) completed scoring using the Jadad's scale [18] for assessing the quality and the risk of bias of the RCTs included. This quantifies the trial quality based on the description and appropriateness of randomization (2 points), blinding procedures ( 2 points), and description of withdrawals ( 1 point). A value less than 3 (over a maximum of 5) usually indicates a lowquality study at high risk of bias [19].

\subsection{Data synthesis and statistical analysis}

All analyses were performed using comprehensive metaanalysis (CMA) 3 and Revman 5.3. Outcomes with at least two studies were meta-analyzed, and in case of only one study, we described the data in a descriptive summary. When multiple assessments were made, the longest follow-up time was included in our analyses.

The primary analysis compared the values of pain scales between participants treated with ALC supplementation vs. controls. We calculated the difference between the means of the treatment and placebo groups using the follow-up data through standardized mean differences (SMD) with their 95\% confidence intervals (CIs), applying a random-effect model [20].

For the secondary analysis, a similar analysis was made for ALC, whilst for ALC/LC this analysis for EMG parameters was limited to the differences between follow-up and baseline values (pre- and 
post-treatment). Both RCTs and descriptive studies reporting quantitative data on EMG were used.

Heterogeneity across studies was assessed by the $\mathrm{I}^{2}$ metric and $\mathrm{Chi}^{2}$ statistics. Given significant heterogeneity $\left(\mathrm{I}^{2} \geq 50 \%, P<0.05\right)$ and for outcomes having at least 5 studies, we planned to run a meta-regression analysis. No sufficient number of studies was reached by any outcome included.

Publication bias was assessed by a visual inspection of funnel plots and calculating the Egger bias test [21]. Then, to account for publication bias, we used the trim-and-fill method, based on the assumption that the effect sizes of all the studies are normally distributed around the center of a funnel plot; in the event of asymmetries, the test adjusts for the potential effect of unpublished studies [21]. For all analyses, a $P$-value less than 0.05 was considered statistically significant.

\section{Results}

\subsection{Search results}

Altogether, the searches yielded 230 non-duplicated articles. After excluding 218 articles based on title/abstract review (predominantly because they did not include participants with diabetic neuropathy), 12 articles were retrieved for full text review. Finally, six articles [22-27] were included in the qualitative synthesis and five [23-27] were included in the quantitative analysis. Of the included articles, three were RCTs [23-25] (Fig. 1).

\subsection{Study and patient characteristics}

Full descriptive details of the included studies are reported in Supplementary Table 1 (ALC-RCTs) and Supplementary Table 2 (LC-descriptive studies). All the studies together included a total of 711 diabetic participants with peripheral neuropathy.

The three RCTs [23-25] included in our meta-analysis included a total of 658 diabetic participants with neuropathy. Across the 3 RCTs, 340 were treated with ALC (mean age $=57.8$ years), whilst the 318 controls (mean age $=57.8$ years) were treated with placebo ( $n=203)$ or methylcobalamine $(n=115)$, as reported in the Supplementary Table 1 . All the three studies included both type 1 and 2 diabetes with a concomitant treatment with oral antidiabetic agents and insulin. The median follow-up period was 48 weeks (range: $24-52$ ). The quality, assessed through the Jadad's scale [18], indicates a low risk of bias.

The other three descriptive studies $[22,26,27]$ reporting data regarding the effect of LC on diabetic neuropathy are described in the Supplementary Table 2. All three descriptive studies were conducted in Turkey and involved 53 people with type 1 and/or 2 diabetes. The median follow-up period for these studies was 8 weeks (range: $2-40$ ).
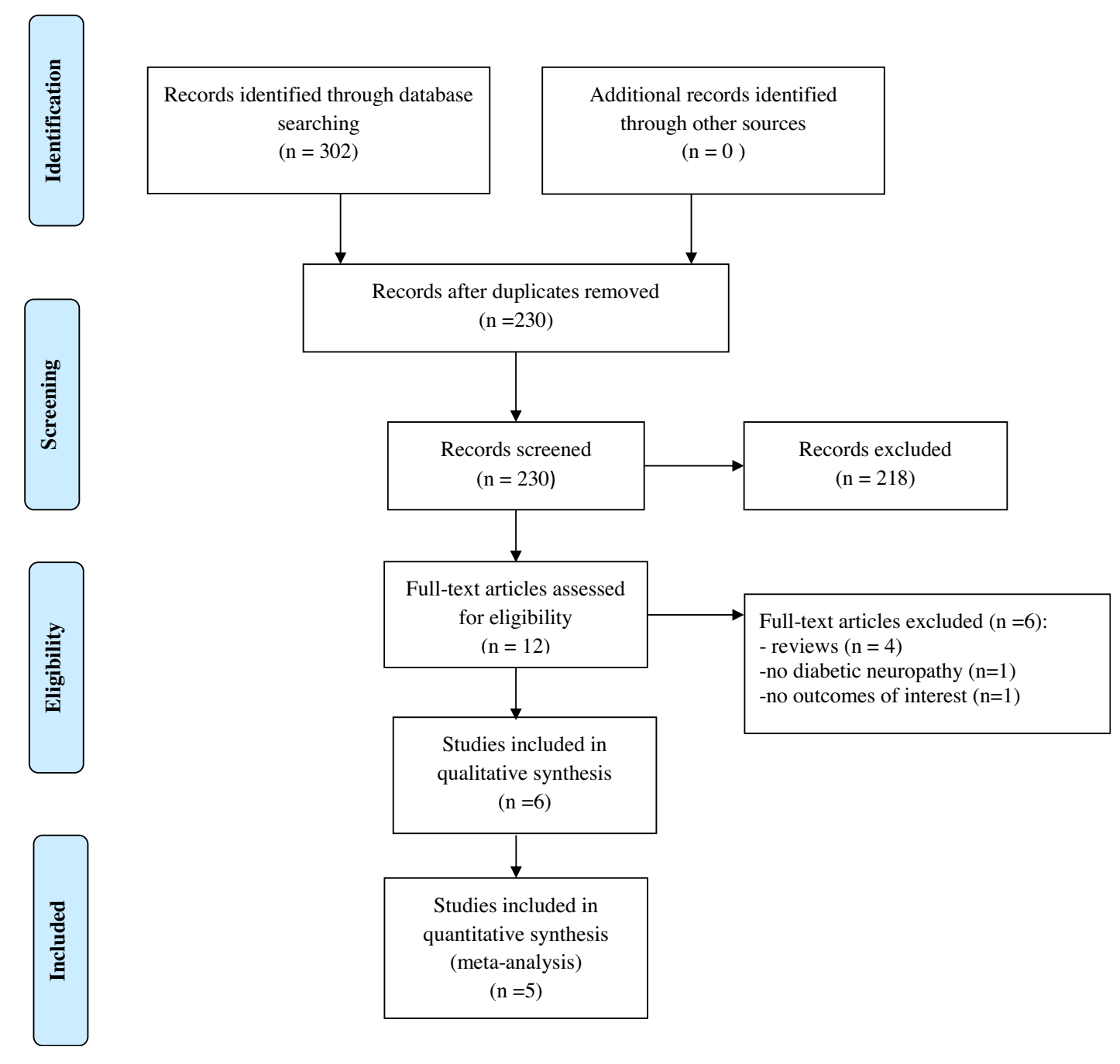

Fig. 1. Prisma flow-chart. 


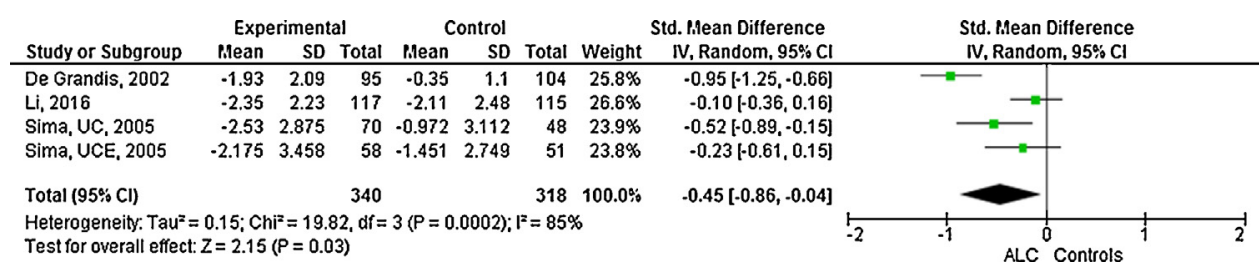

Fig. 2. Effect of acetyl-L-carnitine (ALC) on neuropathic pain in randomized controlled trials. CI: confidence intervals; IV: inverse variance; SD: standard deviations.

\subsection{Meta-analysis of the effect of ALC on pain}

Fig. 2 shows the effect of ALC on pain in people with diabetic neuropathy. Two studies $[23,25]$ reported the pain perception using a visual analogue scale, whilst another RCT [24] used the neuropathy symptom score. Overall, ALC significantly reduced pain perception in 340 participants vs. 318 controls (203 treated with placebo and 115 with methylcobalamine) ( 4 cohorts in 3 RCTs [23-25]; SMD $=-0.45 ; 95 \% \mathrm{CI}:-0.86$ to $-0.04 ; P=0.03 ; \mathrm{I}^{2}=85 \%$ ).

Although the data were heterogeneous, a meta-regression analysis was not possible due to the limited number of the studies available, whilst the evidence of the publication bias was low (Egger bias test $=0.78 ; P=0.48$ ).

\subsection{Meta-analysis of the effect of $A L C$ and $A L C / L C$ on} electromyographic parameters

All studies (except one [22]) reported the effect of ALC/LC on EMG parameters. We were able to meta-analyze pre- and post-test changes from 4 of the 5 studies reporting data on EMG parameters. One study [25] reported the data only as descriptive findings.

Table 1 shows the meta-analysis of EMG parameters between patients treated with ALC vs. controls in two studies [23,24]. Compared to controls, the treatment with ALC improved the nerve conduction velocity a both sensory (SMD $=0.60 ; 95 \% \mathrm{CI}: 0.35-0.86$, $\left.P<0.0001 ; \mathrm{I}^{2}=94 \%\right)$ and motor $(\mathrm{SMD}=0.67 ; 95 \% \mathrm{CI}: 0.43-0.91$, $\left.P<0.0001 ; \mathrm{I}^{2}=0 \%\right)$ ulnar nerve. We observed a similar improvement for both sensory and motor component for ulnar nerve, taking response amplitude as outcome. ALC improved also the response amplitude for motor nerve median as reported in Table 1. Except few exceptions, the heterogeneity was high. The publication bias analysis was not feasible due to the limited number of studies available for each outcome.

Table 2 shows the meta-analysis of EMG parameters calculated as the difference between follow-up and baseline values after ALC/LC treatment. Regarding nerve conduction velocity, ALC/LC significantly improved all the three sensory nerves investigated with a SMD ranging from 0.76 (95\% CI: 0.50 1.02 ; 3 studies; 122 participants) for median nerve to $1.01 \mathrm{~m} / \mathrm{s}$ (95\% CI: 0.74-1.29; 3 studies; 118 participants) for sural nerve. A similar finding was evident for median $(\mathrm{SMD}=0.54 \mathrm{~m} / \mathrm{s}$; 95\% CI: $0.25-0.83 ; 4$ studies; 124 participants) and tibial motor nerve $(\mathrm{SMD}=0.49 \mathrm{~m} / \mathrm{s} ; 95 \% \mathrm{CI}: 0.18-0.79 ; 3$ studies; 86 participants) (Table 2, upper part).

For all these results, the heterogeneity was low (as shown by the $\mathrm{I}^{2}<50 \%$ ) and publication bias was unlikely, except for ulnar motor nerve for which, however, the trim and fill procedure did not change our results.

The effect of ALC/LC on response amplitude seems to be comparable to the effect of nerve conduction velocity. ALC/LC were able to improve the response amplitude for all sensory nerves (with a SMD ranging from 0.79 for sural to $1.04 \mathrm{uV}$ for median nerve) and for all motor nerves (SMD from 0.60 for peroneal to $0.74 \mathrm{mV}$ for tibial), except for median nerve. However, after trimming one study at the left of the mean, also this finding becomes statistically significant $(\mathrm{SMD}=1.46 \mathrm{mV} ; 95 \% \mathrm{CI}$ : $0.24-$ 2.69) (Table 2, lower part).

Contrary to the findings observed for conduction velocity, the heterogeneity for response amplitude was generally high $\left(\mathrm{I}^{2}>70 \%\right)$ for all the outcomes included.

Finally, Sima et al., in their trial [25], reported a not significant effect of ALC on EMG parameters, when compared to placebo, but the data after/before ALC supplementation were not available. Anyway, the trial [25] showed significant improvements in sural nerve fiber numbers and regenerating nerve fiber clusters, and vibration perception was improved.

\subsection{Meta-regression analysis}

Although some outcomes suffered of high heterogeneity (as indicated by an $\mathrm{I}^{2} \geq 50 \%$ ), the number of the studies for each outcome was too low for doing a reliable meta-regression analysis.

Table 1

Electromyographic findings of the studies investigating the role of acetyl-L-carnitine (ALC) compared to controls.

\begin{tabular}{|c|c|c|c|c|c|}
\hline \multirow[t]{2}{*}{ Analysis } & \multicolumn{4}{|c|}{ Meta-analysis } & \multirow{2}{*}{$\frac{\text { Heterogeneity }}{\mathrm{I}^{2}}$} \\
\hline & SMD & $95 \% \mathrm{CI}$ & & $P$-value & \\
\hline \multicolumn{6}{|c|}{ Nerve conduction velocity } \\
\hline $\mathrm{SN}$ median $(\mathrm{m} / \mathrm{s})$ & 0.26 & -0.58 & 1.09 & 0.55 & 80 \\
\hline $\mathrm{SN}$ ulnar $(\mathrm{m} / \mathrm{s})$ & 0.60 & 0.35 & 0.86 & $<\mathbf{0 . 0 0 0 1}$ & 94 \\
\hline SN sural $(\mathrm{m} / \mathrm{s})$ & 0.56 & -0.09 & 1.21 & 0.09 & 75 \\
\hline MN median $(\mathrm{m} / \mathrm{s})$ & 0.45 & -0.20 & 1.10 & 0.18 & 63 \\
\hline MN ulnar $(\mathrm{m} / \mathrm{s})$ & 0.67 & 0.43 & 0.91 & $<0.0001$ & 0 \\
\hline MN peroneal (m/s) & 0.83 & -0.20 & 1.85 & 0.11 & 94 \\
\hline \multicolumn{6}{|l|}{ Response amplitude } \\
\hline SN median (uV) & 0.67 & -0.89 & 2.23 & 0.40 & 93 \\
\hline SN ulnar (uV) & 0.99 & 0.72 & 1.26 & $<\mathbf{0 . 0 0 0 1}$ & 93 \\
\hline SN sural (uV) & 0.71 & -0.66 & 2.07 & 0.31 & 93 \\
\hline MN median (mV) & 0.89 & 0.42 & 1.36 & $<\mathbf{0 . 0 0 0 1}$ & 0 \\
\hline MN ulnar (mV) & 0.74 & 0.46 & 1.03 & $<0.0001$ & 0 \\
\hline MN peroneal (mV) & 0.49 & -0.57 & 1.54 & 0.37 & 85 \\
\hline
\end{tabular}

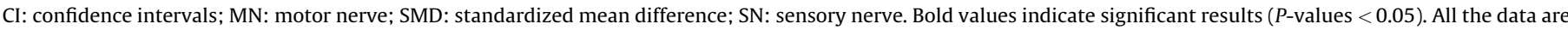
extracted from two studies $[21,22]$. 
Table 2

Electromyographic findings of the studies investigating the role of acetyl-L-carnitine and L-carnitine compared to baseline.

\begin{tabular}{|c|c|c|c|c|c|c|c|c|c|c|}
\hline \multirow[t]{2}{*}{ Analysis } & \multirow{2}{*}{$\begin{array}{l}\text { Number of } \\
\text { studies } \\
\text { (references) }\end{array}$} & \multirow{2}{*}{$\begin{array}{l}\text { Number } \\
\text { participants }\end{array}$} & \multicolumn{4}{|c|}{ Meta-analysis } & \multirow{2}{*}{$\frac{\text { Heterogeneity }}{\mathrm{I}^{2}}$} & \multicolumn{2}{|c|}{ Publication bias } & \multirow{2}{*}{$\begin{array}{l}\text { Classic fail } \\
\text { safe }(n)\end{array}$} \\
\hline & & & SMD & $95 \% \mathrm{CI}$ & & $P$-value & & $\begin{array}{l}\text { Egger bias } \\
\text { and } P \text {-value }\end{array}$ & $\begin{array}{l}\text { Trim and fill }(95 \% \mathrm{CI}) \\
\text { [trimmed] }\end{array}$ & \\
\hline \multicolumn{11}{|c|}{ Nerve conduction velocity } \\
\hline $\mathrm{SN}$ median $(\mathrm{m} / \mathrm{s})$ & $3[21,22,24]$ & 122 & 0.76 & 0.50 & 1.02 & $<\mathbf{0 . 0 0 0 1}$ & 0 & $1.90 ; 0.12$ & $0.66(0.44-0.89)[2]$ & 24 \\
\hline SN ulnar $(\mathrm{m} / \mathrm{s})$ & $3[21,22,24]$ & 119 & 0.89 & 0.53 & 1.26 & $<\mathbf{0 . 0 0 0 1}$ & 44 & $3.22 ; 0.79$ & Unchanged & 31 \\
\hline SN sural $(\mathrm{m} / \mathrm{s})$ & $3[21,22,24]$ & 118 & 1.01 & 0.74 & 1.29 & $<\mathbf{0 . 0 0 0 1}$ & 0 & $-3.10 ; 0.72$ & Unchanged & 38 \\
\hline MN median $(\mathrm{m} / \mathrm{s})$ & $4[21,22,24,25]$ & 124 & 0.54 & 0.25 & 0.83 & $<\mathbf{0 . 0 0 0 1}$ & 18 & $-1.58 ; 0.55$ & $0.45(0.17-0.73)[1]$ & 12 \\
\hline MN ulnar $(\mathrm{m} / \mathrm{s})$ & $4[21,22,24,25]$ & 189 & 0.48 & -0.11 & 1.07 & 0.10 & 85 & $-7.80 ; 0.03$ & Unchanged & 25 \\
\hline MN tibial $(\mathrm{m} / \mathrm{s})$ & $3[22,24,25]$ & 86 & 0.49 & 0.18 & 0.79 & 0.002 & 0 & $1.70 ; 0.05$ & $0.42(0.17-0.66)[2]$ & 5 \\
\hline MN peroneal $(\mathrm{m} / \mathrm{s})$ & $4[21,22,24,25]$ & 192 & 0.62 & -0.27 & 1.50 & 0.17 & 94 & $-9.81 ; 0.29$ & Unchanged & 42 \\
\hline \multicolumn{11}{|l|}{ Response amplitude } \\
\hline SN median $(\mathrm{uV})$ & $3[21,22,24]$ & 102 & 1.04 & 0.12 & 1.95 & 0.03 & 86 & $-2.37 ; 0.76$ & Unchanged & 32 \\
\hline SN ulnar (uV) & $3[21,22,24]$ & 149 & 0.85 & 0.04 & 1.65 & 0.04 & 89 & $-7.98 ; 0.33$ & Unchanged & 40 \\
\hline SN sural (uV) & $4[21,22,24,25]$ & 129 & 0.79 & 0.19 & 1.40 & 0.01 & 81 & $-11.8 ; 0.11$ & Unchanged & 37 \\
\hline MN median (mV) & $4[21,22,24,25]$ & 90 & 1.09 & -0.16 & 2.33 & 0.09 & 93 & $5.01 ; 0.72$ & $1.46(0.24-2.69)[1]$ & 36 \\
\hline MN ulnar $(\mathrm{mV})$ & $4[21,22,24,25]$ & 156 & 0.72 & 0.26 & 1.19 & 0.002 & 71 & $-5.12 ; 0.14$ & Unchanged & 36 \\
\hline MN tibial (mV) & $3[22,24,25]$ & 91 & 0.74 & 0.11 & 1.34 & 0.02 & 74 & $-8.59 ; 0.23$ & Unchanged & 16 \\
\hline MN peroneal $(\mathrm{mV})$ & $4[21,22,24,25]$ & 125 & 0.60 & 0.04 & 1.16 & 0.04 & 76 & $1.50 ; 0.76$ & Unchanged & 15 \\
\hline
\end{tabular}

CI: confidence intervals; MN: motor nerve; SMD: standardized mean difference; SN: sensory nerve. Bold values indicate significant results $(P$-values $<0.05)$.

\subsection{Adverse events}

As showed in the Supplementary Table 3, no severe side effects (e.g. death, onset of cardiovascular diseases) emerged after treatment with ALC/LC in RCTs and in one study [25], some neurological signs (pain, hyperesthesia and paraesthesia) were more frequent in placebo group than ALC. All the studies, however, reported that people treated with ALC/LC experienced some gastrointestinal side effects of minor entity, like vomiting or nausea.

\section{Discussion}

In this systematic review and meta-analysis including for pain, three RCTs and three observational studies and for EMG two studies for a total of 711 diabetic participants, we showed that ALC could have a beneficial effect on pain and electromyographic parameters in diabetic people with peripheral neuropathy compared to controls, whilst the treatment with ALC/LC was able to improve EMG parameters compared to baseline.

Compared to the control group, ALC was associated with a significant reduction in pain perception in people affected by diabetic neuropathy. The mechanism leading to this analgesic effect seems to be related to the acetylation and the activation of the transcription of NF-kB, promoted by ALC, acting as a donor of acetyl groups $[12,28]$. This activation corresponds to an increase in the expression of mGlu-2 receptor that link glutamate in the synapses of the dorsal horn with a consequent reduction in pain hypersensitivity $[12,28]$. As previously reported in the metaanalysis of Li et al. [15], the effect of ALC on neuropathic pain was stronger in diabetes than other conditions. One hypothesis is that in diabetes, the effect of glutamate is more pronounced than other neuropathies $[29,30]$ and consequently, ALC works better in this condition.

The descriptive studies reported that LC is able to improve EMG parameters, confirming that, beyond the activity of ALC as a donor of acetyl groups, carnitine supplementation anyway presents a neurotrophic effect. The reasons of why some nerves have and other have not a beneficial effect from LC is not known and further research is needed to disentangle this issue.

In our meta-analysis, ALC/LC were also able to significantly improve the nerve conduction velocity and the amplitude of several upper and lower limbs nerves. In EMG, latency is the time needed for the stimulus to initiate an evoked potential: this parameter reflects the conduction along the fastest fibers. Peak latency is the latency along the majority of axons and is measured at the peak amplitude. Both these parameters are affected by the state of the myelination of the nerve [31] Reduction of amplitudes of recorded responses generally indicates a loss of axons [31]. The conduction velocity also depends on the state of myelination and is often decreased in disorders affecting nerve myelination, although it could be normal if a few myelinated axons remain intact [31]. From a molecular point of view, ALC seems to able to enhance the expression of NGF and its receptors, increases the regeneration of the nerve reducing the myelinic degeneration and finally improves the anatomy and physiology of the nerve $[8,14,32]$. All these factors probably contributed to the effect of ALC on EMG parameters.

The benefits of ALC treatment in diabetic patient are amplified by the fact there are to date very few adverse events reported in the literature. All the three RCTs [23-25], in fact, reported a similar frequency of side effects among people treated with ALC and controls, whilst the only descriptive study [26] reporting adverse events showed that only 2 patients were affected by an adverse effect and they did not discontinue the treatment with this supplement.

The findings of our work should be interpreted within its limitations. First, the findings of EMG between treated and control group were substantially limited only to two studies. Thus, we were not able to run a reliable meta-regression analysis to verify if the improvement in the EMG parameters was associated with decrease in pain. Other trials are needed to disentangle this issue. Second, most of all the amplitude parameters and the primary outcome suffered from high heterogeneity, and we were not able the address this heterogeneity. Finally, the length of follow-up of all the studies included was relatively short and future studies with a longer follow-up period are needed. Similarly, the mean dosage of ALC/LC was highly variable among the studies. Among the strengths of our work, we can consider that is the first metaanalysis investigating the effect of ALC on pain and EMG parameters on diabetic neuropathy. Moreover, our review suggests that ALC treatment is well-tolerated in diabetic patients.

In conclusion, our work suggests that ALC may be effective in reducing pain due to diabetic neuropathy compared to active or placebo controls. Moreover, ALC seems to improve some EMG parameters for both sensory and motor nerves compared to controls and similarly to LC compared to baseline estimates. Future RCTs are needed confirm our findings, particularly regarding the EMG parameters compared to placebo. 


\section{Authorship}

All authors have made substantial contributions to all of the following: (1) the conception and design of the study, or acquisition of data, or analysis and interpretation of data; (2) drafting the article or revising it critically for important intellectual content; (3) final approval of the version to be submitted.

\section{Funding source}

This research received an unrestricted grant from Sigma-Tau. The sponsor did not have any role in the study design; in the collection, analysis and interpretation of data; in the writing of the report; and in the decision to submit the article for publication.

\section{Disclosure of interest}

The authors declare that they have no competing interest.

\section{Appendix A. Supplementary data}

Supplementary data associated with this article can be found, in the online version, at http://dx.doi.org/10.1016/j.eurger.2017.01. 002 .

\section{References}

[1] Pirart J. Diabetes mellitus and its degenerative complications: a prospective study of 4400 patients observed between 1947 and 1973. Diabetes Care 1978;1:252-63. http://dx.doi.org/10.1016/j.pain.2007.08.033.

[2] Benbow SJ, Wallymahmed ME, MacFarlane I. Diabetic peripheral neuropathy and quality of life. QJM 1998;91:733-7. http://dx.doi.org/10.1093/qjmed/ 91.11.733.

[3] Boulton AJM. Treatments for diabetic neuropathy. Curr Diab Rep 2001;1:12732. http://dx.doi.org/10.1007/s11892-001-0024-8.

[4] Griebeler ML, Tsapas A, Brito JP, Wang Z, Phung OJ, Montori VM, et al Pharmacologic interventions for painful diabetic neuropathy: an umbrella systematic review and comparative effectiveness network meta-analysis (Protocol). Syst Rev 2012;1:61. http://dx.doi.org/10.1186/2046-4053-1-61.

[5] Faradji V, Sotelo J. Low serum levels of nerve growth factor in diabetic neuropathy. Acta Neurol Scand 1990;81:402-6.

[6] Levine J, Panchalingam K, McClure RJ, Gershon S, Pettegrew JW. Effects of acetyl-L-carnitine and myo-inositol on high-energy phosphate and membrane phospholipid metabolism in zebra fish: a 31P-NMR-spectroscopy study. Neurochem Res 2003;28:687-90.

[7] Traina G, Federighi G, Macchi M, Bernardi R, Durante M, Brunelli M. Modulation of myelin basic protein gene expression by acetyl-L-carnitine. Mol Neurobiol 2011;44:1-6. http://dx.doi.org/10.1007/s12035-011-8189-x.

[8] Vivoli E, Di Cesare Mannelli L, Salvicchi A, Bartolini A, Koverech A, Nicolai R, et al. Acetyl-l-carnitine increases artemin level and prevents neurotrophic factor alterations during neuropathy. Neuroscience 2010;167:1168-74. http://dx.doi.org/10.1016/j.neuroscience.2010.03.017.

[9] Vanotti A, Osio M, Mailland E, Nascimbene C, Capiluppi E, Mariani C. Overview on pathophysiology and newer approaches to treatment of peripheral neuropathies. CNS Drugs 2007;21(Suppl. 1.). http://dx.doi.org/10.2165/ 00023210-200721001-00002.

[10] Traina G. The neurobiology of acetyl-L-carnitine. Front Biosci (Landmark Ed) 2016;21:1314-29.
[11] Ido Y, McHowat J, Chang KC, Arrigoni-Martelli E, Orfalian Z, Kilo C, et al. Neural dysfunction and metabolic imbalances in diabetic rats: prevention by acetyl-L-carnitine. Diabetes 1994;43:1469-77. http://dx.doi.org/10.2337/diabetes.43.12.1469.

[12] Chiechio S, Copani A, Iv RWG, Nicoletti F. Acetyl-L-carnitine in neuropathic pain. CNS Drugs 2007;21(1):31-8.

[13] Malaguarnera M. Carnitine derivatives: clinical usefulness. Curr Opin Gastroenterol 2012;28:166-76. http://dx.doi.org/10.1097/MOG.0b013e3283505a3b.

[14] Watson JC, Dyck PJB. Peripheral neuropathy: a practical approach to diagnosis and symptom management. Mayo Clin Proc 2015;90:940-51. http:// dx.doi.org/10.1016/j.mayocp.2015.05.004.

[15] Li S, Li Q Li Y, Li L, Tian H, Sun X. Acetyl-L-carnitine in the treatment of peripheral neuropathic pain: a systematic review and meta-analysis of randomized controlled trials. Plos One 2015;10:1-10. http://dx.doi.org/10.1371/ journal.pone.0119479.

[16] Liberati A, Altman DG, Tetzlaff J, Mulrow C, Gøtzsche PC, Ioannidis JPA, et al. The Prisma statement for reporting systematic reviews and meta-analyses of studies that evaluate health care interventions: explanation and elaboration. Plos Med 2009;6:e1000100. http://dx.doi.org/10.1371/journal.pmed.1000100.

[17] Stroup DF, Berlin J, Morton SC, Olkin I, Williamson GD, Rennie D, et al. Metaanalysis of observational studies in epidemiology: a proposal for reporting. JAMA 2000;283:2008-12. http://dx.doi.org/10.1001/jama.283.15.2008.

[18] Jadad AR, Moore RA, Carroll D, Jenkinson C, Reynolds DJ, Gavaghan DJ, et al. Assessing the quality of reports of randomized clinical trials: is blinding necessary? Control Clin Trials 1996;17:1-12.

[19] Jadad AR. The merits of measuring the quality of clinical trials: is it becoming a Byzantine discussion? Transpl Int 2009;22:1028. http://dx.doi.org/10.1111/ j.1432-2277.2009.00919.x.

[20] DerSimonian R, Laird N. Meta-analysis in clinical trials. Control Clin Trials 1986;7:177-88. http://dx.doi.org/10.1016/0197-2456(86)90046-2.

[21] Egger M, Davey Smith G, Schneider M, Minder C. Bias in meta-analysis detected by a simple, graphical test. BMJ 1997;315:629-34. http:/ dx.doi.org/10.1136/bmj.316.7129.469.

[22] Cakir N, Yctkin III, Karako A, Karakan T. L-Carnitine in the treatment of painful diabetic neuropathy and its effect on plasma [3-endorphin levels 2000;61: 871-6.

[23] De Grandis D, Minardi C. Acetyl-L-carnitine (levacecarnine) in the treatment of diabetic neuropathy. A long-term, randomised, double-blind, placebo-controlled study. Drugs R D 2002;3:223-31.

[24] Li S, Chen X, Li OQ Du J, Liu Z, Peng Y, et al. Effects of acetyl-L-carnitine and methylcobalamin for diabetic peripheral neuropathy: a multicenter, randomized, double-blinded, controlled trial. J Diabetes Investig 2016. doi:10.1111/ jdi.12493.

[25] Sima AAFAF, Calvani M, Mehra M, Amato A Acetyl-L-carnitine improves pain, nerve regeneration, and vibratory perception in patients with chronic diabetic neuropathy: an analysis of two randomized placebo-controlled trials. Diabetes Care 2005;28:89-94. http://dx.doi.org/10.2337/diacare.28.1.89.

[26] Ulvi H, Aygül R, Demir R. Effect of L-carnitine on diabetic neuropathy and ventricular dispersion in patients with diabetes mellitus. Turkish J Med Sci 2010;40:169-75. doi:10.3906/sag-0807-7.

[27] Uzun N, Sarikaya S, Uluduz D, Aydin A. Peripheric and automatic neuropathy in children with type 1 diabetes mellitus: the effect of L-carnitine treatment on the peripheral and autonomic nervous system. Electromyogr Clin Neurophysiol 2005;45:343-51.

[28] Onofrj M, Ciccocioppo F, Varanese S, di Muzio A, Calvani M, Chiechio S, et al. Acetyl-L-carnitine: from a biological curiosity to a drug for the peripheral nervous system and beyond. Expert Rev Neurother 2013;13:925-36. http:/l dx.doi.org/10.1586/14737175.2013.814930.

[29] Anjaneyulu M, Berent-Spillson A, Russell JW. Metabotropic glutamate receptors (mGluRs) and diabetic neuropathy. Curr Drug Targets 2008;9:85-93.

[30] Davalli AM, Perego C, Folli FB. The potential role of glutamate in the current diabetes epidemic. Acta Diabetol 2012;49:167-83. http://dx.doi.org/10.1007/ s00592-011-0364-Z.

[31] Aminoff MJ. Electrodiag Clin Neurol 2005. doi:10.1016/B978-0-443-066474.50016-1.

[32] Taglialatela G, Navarra D, Cruciani R, Ramacci MT, Alemà GS, Angelucci L Acetyl-L-carnitine treatment increases nerve growth factor levels and choline acetyltransferase activity in the central nervous system of aged rats. Exp Gerontol 1994;29:55-66. 